\title{
The death of a genre: self-driving cars and car racing video games
}

\author{
Jens Dobberthin ${ }^{1}$, Jian $\mathrm{Wu}^{2}$ \\ Fraunhofer Application Center KEIM, Esslingen, Germany ${ }^{1}$ \\ Faculty of Information Technology, Hochschule Esslingen University of Applied Sciences, \\ Germany $^{2}$
}

\begin{abstract}
Whilst self-driving cars have a long tradition in literature and movies, the role of self-driving cars in video games is quite unclear. The application presented in this paper is an early attempt to develop a car racing video game in the age of self-driving cars. Most likely, self-driving cars will cause the death of this genre, and thus our demo is meant as a thought-provoking contribution.
\end{abstract}

\section{Introduction}

Over many decades, car racing video games have attracted a lot of players mainly because of their simple game mechanics and obvious playing goals. Innovation in this game genre was particularly driven by improvements of existing features. Hence, better graphics, realistic driving physics, or non-driving related refinements such as editable engine characteristics or a career mode, were introduced to create more realistic games (Sterbenz 2011, p. 53). Yet, no development has ever resulted in a breaking through concept which has changed the genre.

Game genres can be considered as a set of conventions and as an easy way for game producers and distributors to clearly communicate about what a potential player can expect from a game (Beil 2013, p. 39). Though some games could fit into two or more genres it is unquestionable, that the primary goal of a car racing video game is to steer a car on a track. However, this simple assumption might be wrong in the future.

Now, we are at the advent of an era that will change our understanding of cars in reality as well as in video games. Self-driving cars are expected to be deployed in cities by the year 2030 (Dokic et al. 2015). This fact instantly raises many questions. What is the purpose of a car racing video game, when in reality non-driving-related activities outweigh drivingrelated-activities? What will happen to the genre when players can only adjust their destination? 
What if nobody can remember how to drive a car any longer? Will this be the death of a whole genre?

\section{Context}

As part of their curriculum computer science students were asked to form project teams and to develop a piece of software in close collaboration with companies, research institutes and other public organizations. The two main goals were, firstly, to foster team work and, secondly, to experience software development in the real world.

A group of four students took up the challenge to develop the next evolution of a car racing video game. Just a few basic conditions were set. Firstly, the game should tackle current research areas of the Fraunhofer KEIM, namely, electromobility and autonomous driving. Secondly, the game should address risks resulting from these new technologies. Finally, the game should work on standard hardware and should be published under an open source licence.

\section{Gameplay}

During the game players perform certain actions. These actions are embedded in a rather conventional gameplay. To start the game players choose a car with specific characteristics, for example, a car with a combustion engine or an electric vehicle. Whilst on the road players cannot steer or accelerate the vehicle as in traditional games. Instead they interact in different minigames. An example of such minigames is a cyber-attack, which can only be counterattacked by solving a puzzle. In this special minigame, the vulnerability of highly interconnected cars is addressed. Eventually, after passing the goal line players are invited to key in their names to get listed in the hall of fame.

\section{Conclusion}

Self-driving cars have gained much attention in literature and movies (Kröger 2015). However, considering video games as the defining medium of digital culture (Freyermuth 2015 , p. 99), it is astonishing that, to the best of our knowledge, the topic has not been addressed so far in video games.

The survival of car racing video games depends first and foremost on the ability to entertain gamers. It is not quite clear how future car racing games will address this problem. With the advent of self-driving cars there is the fear that a whole genre might disappear. The demonstrated application (Car Racing Game 2015) aimed to provide an early outlook on the future of car racing video games. 


\section{Acknowledgements}

The authors would like to thank the students Oliver Feucht, Konstantin Wacker, Tobias Klingel und Patrick Quell for their enthusiastic team work.

\section{Contact}

Jens Dobberthin / Jian Wu

Fraunhofer Anwendungszentrum KEIM, Flandernstraße 101, 73732 Esslingen am Neckar jens.dobberthin@iao.fraunhofer.de / jian.wu@hs-esslingen.de

\section{References}

Beil, B. (2013). Game Studies: eine Einführung. Berlin: Lit.

Dokic, J., Meyer, G. \& Müller, B. (2015). European Roadmap Smart Systems for Automated Driving (No. 1.2). EPoSS. http://www.smart-systems-integration.org/public/documents/publications

Freyermuth, G. S. (2015). Games | Game Design | Game Studies: Eine Einführung. Bielefeld: transcript Verlag.

Kröger, F. (2015). Das automatisierte Fahren im gesellschaftsgeschichtlichen und kulturwissenschaftlichen Kontext, in: Maurer, M., Gerdes, J.C., Lenz, B. \& Winner, H. (Eds.): Autonomes Fahren. Berlin: Springer Verlag. pp. 41-67.

Sterbenz, B. (2011). Zur Theorie der Computerspiel-Genres. Diplomarbeit. Universität Wien. Philologisch-Kulturwissenschaftliche Fakultät. http://othes.univie.ac.at/13565/

Car Racing Game. (2015). Sources available at: https://gitlab.com/jedob/car-racing-2030 
